Revue d'histoire de l'Amérique française

REYUE D.HISTOIRE DE L'AMÉRIQUE FRANÇAISE

\title{
COMMISSION DES BIENS CULTURELS, Les chemins de la mémoire, 3 : Biens mobiliers du Québec (Sainte-Foy, Les Pulblications du Québec, 1999), 428 p.
}

\section{Paul-Louis Martin}

Volume 55, numéro 2, automne 2001

URI : https://id.erudit.org/iderudit/010369ar

DOI : https://doi.org/10.7202/010369ar

Aller au sommaire du numéro

Éditeur(s)

Institut d'histoire de l'Amérique française

ISSN

0035-2357 (imprimé)

1492-1383 (numérique)

Découvrir la revue

Citer ce compte rendu

Martin, P.-L. (2001). Compte rendu de [COMMISSION DES BIENS CULTURELS, Les chemins de la mémoire, 3 : Biens mobiliers du Québec (Sainte-Foy, Les Publications du Québec, 1999), 428 p.] Revue d'histoire de l'Amérique française, 55(2), 257-261. https://doi.org/10.7202/010369ar d'utilisation que vous pouvez consulter en ligne.

https://apropos.erudit.org/fr/usagers/politique-dutilisation/ 
jusqu'à un certain point l'habileté du lecteur à répondre de manière satisfaisante et éclairée. Par exemple, j'ose émettre l'hypothèse que des facteurs reliés à l'importance donnée par la population à la formation universitaire ou d'autres reliés à la culture politique des infirmières du Québec puissent avoir joué un rôle important dans les questions reliées à l'éducation. Similairement, il est urgent de se pencher sur l'histoire des tensions entre l'Ordre des infirmières et infirmiers du Québec et l'Association des infirmières et infirmiers du Canada. L'étude de ces sujets ajouterait certainement des éléments utiles à l'analyse et il est à espérer que l'auteure se penchera sur ces aspects lors de prochains travaux.

Il est pertinent de souligner que tout au long du volume, de courtes biographies d'individus ayant joué des rôles de premier plan sont offertes au lecteur. On y retrouve d'importantes données qui facilitent grandement la lecture du texte. En conclusion, il m'apparaît évident que l'ouvrage de Cohen est de qualité, qu'il ajoute significativement aux connaissances sur l'histoire de la profession infirmière au Québec et qu'il peut mener à de nouveaux questionnements.

PAULINE PAUL

Faculté de Nursing Université de l'Alberta

COMMISSION DES BIENS CULTURELS, Les chemins de la mémoire, 3 : Biens mobiliers du Québec (Sainte-Foy, Les Publications du Québec, 1999), 428 p.

Ce volume consacré aux biens mobiliers fait suite aux deux premiers tomes parus en 1990 et 1991 et qui présentaient les immeubles, les sites et les arrondissements que le Québec protège légalement en vertu de sa Loi sur les biens culturels. Réalisés à l'initiative et sous l'égide de la Commission des biens culturels, les trois volumes de fort belle tenue permettent de diffuser adéquatement une meilleure connaissance des principaux trésors du patrimoine collectif.

Une quarantaine d'auteurs et de spécialistes participent à ce troisième volume qui présente trois types de biens mobiliers. Les deux tiers de l'ouvrage sont consacrés aux œuvres et objets d'art. Suivent les descriptions de quatorze fonds d'archives et de documents, conservés pour la plupart dans les institutions d'enseignement et, en dernière partie, plutôt maigre celle-là, diverses collections d'objets domestiques, de meubles, d'outils de métier, un violon Stradivarius, des poissons fossilisés et un drapeau fleur- 
delisé. Ce déséquilibre évident entre les familles d'objets et de biens mobiliers, pour ne pas dire le caractère apparemment hétéroclite de l'ensemble, peut surprendre le lecteur. Il mérite certes une explication. C'est que le recours à la loi est tout à fait aléatoire, discrétionnaire et essentiellement conjoncturel; il ne correspond à aucune planification stratégique de conservation préventive, ni de reconnaissance publique de la valeur intrinsèque des biens culturels, pas plus meubles qu'immeubles. Il aurait d'ailleurs été fort éclairant de connaitre la petite histoire de l'attribution de certains statuts : ici, pour contrer la menace d'une "modernisation" aveugle ou les agissements d'un curé brocanteur, là, pour faciliter l'octroi de crédits publics à la restauration, là encore pour préserver l'intégralité d'un ensemble ou carrément pour éviter l'exportation. L'essentiel, se diton, est que ces biens aient été préservés, mais il n'empêche qu'une approche plus active et moins velléitaire de la part de l'État serait plus que jamais souhaitable en matière de patrimoine. C'est ce qu'ont répété récemment de nombreux groupes associatifs devant le Comité Arpin, dont le rapport, déposé en novembre 2000, doit inspirer une politique du patrimoine, qui se fait cependant toujours attendre.

$\mathrm{Au}$ sens strictement juridique du terme, une assez grande partie des œuvres d'art religieux décrites dans ce volume ne sont plus des objets mobiliers, elles sont devenues des immeubles par destination, attachés «à fer et à clou " aux structures architecturales : retables, lambris de murs, baldaquins, bancs d'œuvre, peintures marouflées, vitraux, chaires, portes de sacristie, buffets d'orgue et autres éléments font bel et bien partie d'un ensemble ornemental que l'on n'a commencé à protéger comme tel qu'en 1957. Citons entre autres les décors des églises de L’Islet, de Neuville, de Saint-Pierre de l'Île-d'Orléans puis, quelques années plus tard, ceux de Saint-Sulpice, de Saint-Elzéar de Saint-Jean-Baptiste-de Rouville et de Sault-au-Récollet qui révèlent une pratique de conservation beaucoup plus sensée qu'une intervention à la pièce, isolée de son cadre architectural. Bien sûr, des circonstances obligent parfois à protéger un élément séparé, mais c'est souvent au détriment de la compréhension de l'ensemble du décor d'une église et même du génie de l'artiste.

La Commission des biens culturels a recruté les meilleurs historiens de l'art et les spécialistes des différents domaines. Ceux-ci réussissent à renouveler les approches descriptives et à susciter l'intérêt du lecteur. Au-delà des objets et de leur valeur artistique ou historique indéniable se profile en effet souvent l'esprit et l'atmosphère d'une époque, faite de ferveur religieuse et de générosité des fidèles. En témoigne par exemple le pro- 
gramme iconographique de l'ensemble des six statues de l'église de Deschambault. L'auteur du texte, Mario Béland, fait bien voir le caractère exceptionnel de ces œuvres, réalisées vers 1820 par les architectes et sculpteurs Baillargé, père et fils, en plus d'évoquer un contexte d'acquisition illustrant nettement la volonté d'embellissement et l'engagement des paroissiens à l'égard de leur église. La petite histoire de la colossale statue de Notre-Dame du Saguenay est tout aussi révélatrice du sentiment religieux et de la piété qui s'expriment jusque dans les paysages à la fin du $\mathrm{xIx}^{\mathrm{e}}$ siècle. Mesurant 8,5 mètres de hauteur et pesant plusieurs tonnes, cette ouvre de Louis Jobin a été hissée en juillet 1881 au sommet du Cap Trinité : il a fallu vingt-deux étapes pour franchir le dernier promontoire de deux cents mètres, à l'aide de palans, de billes et de plans inclinés. Une cérémonie fastueuse entoure la bénédiction de la statue, le 15 septembre suivant, sous la présidence de l'instigateur du projet, $\mathrm{M}^{\mathrm{gr}}$ Dominique Racine, évêque de Chicoutimi. Depuis lors, l'imposante statue de la Vierge Marie marque le paysage saguenayen et contribue à ses attraits touristiques.

Ce ne sont là que quelques exemples des textes fort instructifs et éclairants qui composent ce volume : il faudrait aussi citer la contribution de René Villeneuve qui décrit avec brio le trésor exceptionnel de NotreDame-de-Lorette, fait de pièces d'orfèvrerie, d'ornements sacerdotaux, de parements brodés, de sculptures et d'objets mobiliers. Citer encore les nombreux textes de Laurier Lacroix, en particulier son analyse de l'œuvre picturale d'Ozias Leduc dans l'église Notre-Dame-de-la-Présentation, à Shawinigan-Sud. Dernier grand épisode d'une tradition ornementale plusieurs fois séculaire, cette œuvre originale parvient à intégrer à l'espace sacré le travailleur des champs et l'ouvrier d'usine marquant ainsi le changement profond que vit le Québec artistique des années 1940, sinon son entrée dans la modernité.

Les ensembles de meubles et d'objets domestiques conservés dans leur cadre d'origine, autrement dit "in situ», sont extrêmement rares au Québec. Pour diverses raisons, les réflexes de conservation intégrale des intérieurs domestiques n'ont guère été valorisés, cédant le pas aux attraits de la nouveauté et de la modernisation du cadre de vie. Deux collections ont heureusement échappé au courant dominant, soit celui de la résidence de l'architecte montréalais Ernest Cormier et celui de la maison StuartHenry, à Québec. Robert Little présente d'abord le parcours et les sources d'inspiration du célèbre architecte, auteur des édifices les plus prestigieux de la période Art Déco. Il nous entraîne ensuite à l’intérieur de sa résidence où se déploie dans l'espace et jusque dans les moindres objets tout 
le talent de ce remarquable créateur. Malgré l'absence de photographies en couleur, on perçoit dans ce cadre de vie la recherche patiente de la pureté des lignes, de la sobriété des couleurs et des formes dépouillées. Cormier a même poussé le raffinement jusqu'à importer du Japon des papiers tentures fabriqués avec l'écorce de prunier. Il s'agit ici d'un ensemble absolument exceptionnel, d'un véritable trésor patrimonial apte à émouvoir et à faire comprendre la riche personnalité d'un grand artiste. Il faut souhaiter voir se réaliser un jour le projet de musée que porte la rumeur publique, afin de rendre accessible non seulement l'univers intime du dernier propriétaire de cette résidence unique, Pierre Elliott Trudeau, mais aussi le cheminement et le cadre de vie de ce concepteur et architecte de renommée internationale.

La collection Stuart-Henry illustre de son côté le mode de vie de la bourgeoisie anglo-saxonne de la ville de Québec, dans la seconde moitié du $\mathrm{XIX}^{\mathrm{e}}$ siècle et au début du $\mathrm{xx}^{\mathrm{e}}$. Au-delà de la description de l'ameublement de styles Adam, Empire, Sheraton ou néo-renaissance et des nombreuses collections de porcelaines anglaises ou chinoises, l'auteure du texte, Claire Desmeules, réussit à évoquer le quotidien et les occupations d'une famille représentative de son époque : les petits objets et les souvenirs personnels servent ainsi à illustrer les usages de politesse et de bienséance entourant les loisirs et les relations sociales. La mise en valeur «in situ » de cet ensemble y gagne dès lors en profondeur et justifie la protection légale de la collection.

Le statut légal accordé aux quatorze fonds d'archives soulève plusieurs interrogations. Pourquoi reconnaître telle collection déjà conservée par une institution ou figurant dans un dépôt d'archives? L'archiviste Gilles Héon répond à la question en expliquant en introduction à ce chapitre que le recours à la loi sert ici à distinguer la valeur exceptionnelle d'un fonds et à marquer sa grande valeur historique. L'objectif des archivistes, fort louable, s'accompagne d'une stratégie visant une couverture territoriale et thématique, apte à susciter intérêt et fierté chez les conservateurs et les gardiens de la mémoire. L'espace manque pour décrire la richesse du contenu des fonds de l'écrivain Jacques Ferron, de l'historien Lionel Groulx, du patriote Jean-Joseph Girouard, de l'industriel J.-E.-Alfred Dubuc, des fondateurs de l'Université Bishop et de plusieurs autres figures importantes. On comprend que la reconnaissance légale soit d'autant plus justifiée que l'État multiplie dorénavant les partenaires de la conservation, par la voie de l'agrément des dépôts d'archives, et qu'il importe de signaler de façon tangible autant que symbolique l'importance de ces trésors documentaires. 
Amorcée il y a quatorze ans, l'entreprise de publication des «Chemins de la mémoire» trouve ainsi sa conclusion. Le grand public peut accéder facilement au trésor commun des patrimoines matériels et les spécialistes peuvent y puiser les sources, les références et les opinions critiques qui alimenteront les recherches de demain. Il convient à cet égard de saluer la ténacité et la constance de la Commission des biens culturels.

PAUL-LOUIS MARTIN Département des sciences humaines Université du Québec à Trois-Rivières

CURTIS, Bruce, The Politics of Population. State Formation, Statistics, and the Census of Canada, I840-I 875 (Toronto, University of Toronto Press, 200 I), xi-385 p.

Depuis maintenant plus de 15 ans, Bruce Curtis a apporté à l'historiographie canadienne les méthodes et les problématiques de la sociologie historique. Ses travaux sont un remarquable mélange de recherche empirique soigneuse et de sophistication théorique. Son dernier ouvrage ne dément nullement cette marque de commerce.

Paradis quantitatif des historiens de la socio-économie, les recensements tenus au Canada depuis le milieu du xix ${ }^{\mathrm{e}}$ siècle n'avaient jamais fait l'objet d'une étude systématique. C'est à cette tâche que Curtis s'attache en prenant pour objet les recensements canadiens de 1840 à 1875. L'objectif n'est pas tant de faire une critique exhaustive de ce type de source (quoique plusieurs passages permettent de relativiser sa pertinence heuristique et de mettre en garde contre la «naïvely realist attitude» (p. 16) des historiens en la matière) que d'étudier les recensements comme un processus de construction de l'État canadien.

La perspective de fond prise par Curtis dans l'analyse des recensements repose sur un principe de base : «Census are made, not taken» (p. 33). L'acte de recenser, de compter les populations implique un processus de catégorisation et de généralisation qui est tout à la fois et en même temps un acte politique. En ce sens, le recensement est un «investissement» (au sens du sociologue Thévenot), soit une prise de possession par un savoir donné et un ordonnancement particulier des rapports sociaux. On comprendra que toute l'analyse sera axée à la fois sur les rapports de savoir/pouvoir au cœur du processus de collecte des données et sur la validation scientifique de ce processus. En fait, la justification scientifique sous-jacente au geste de recenser fait de celui-ci une «boîte noire» qui en masque la dimension pro- 